\title{
MECANISMOS MOLECULARES DE ACCÃO ANTIOXIDANTE DE VIAS CELULARES INTRÍNSECAS E DO RESVERATROL
}

\author{
Patrícia de Almeida Machado ${ }^{1}, V^{2}$ Vanessa de Sousa Cruz Pimenta ${ }^{2}$, Eugênio \\ ${\text { Gonçalves de } \text { Araújo }^{3} \text {, Gabriela Hadler Gabriel }}^{4}$ \\ 1 Mestranda do Programa de Pós-Graduação em Ciência Animal da Escola de Veterinária e \\ Zootecnia da Universidade Federal de Goiás (patriciadealmeidamachado@hotmail.com.br) Goiás- \\ Brasil \\ 2 Pós doutoranda, Laboratório Multiusuário de Cultivo Celular da UFG \\ 3 Prof. Dr. do Setor de Patologia Veterinária da Universidade Federal de Goiás \\ 4 Mestranda do Programa de Pós-Graduação em Ciência Animal da Escola de Veterinária e \\ Zootecnia da Universidade Federal de Goiás
}

Recebido em: 08/04/2016 - Aprovado em: 30/05/2016 - Publicado em: 20/06/2016 DOI: 10.18677/Enciclopedia_Biosfera_2016_065

\begin{abstract}
RESUMO
O aumento intracelular de ROS induz o estresse oxidativo, que pode desencadear a morte celular por apoptose, por ativação de cascatas apoptóticas intrínsecas. O estresse oxidativo pode se apresentar no organismo em diferentes situações e nos mais diversos órgãos e tecidos. O consumo de dieta rica em gordura está relacionado com o aumento do estresse oxidativo e disfunções mitocondriais em diversos órgãos. As substâncias antioxidantes atuam inibindo a produção excedente de espécies reativas de oxigênio (ROS), eliminam radicais livres e consequentemente diminuem a apoptose. O resveratrol é um composto que ocorre naturalmente em plantas e tem demonstrado possuir diversas propriedades biológicas, dentre elas a atividade antioxidante. Com esta revisão de literatura pretendeu-se reunir informações sobre os mecanismos moleculares de ação antioxidante de vias celulares intrínsecas e do resveratrol nas células.
\end{abstract}

PALAVRAS-CHAVE: Diabete, infarto, isquemia.

\section{MECHANISMS OF ROADS ANTIOXIDANT ACTION MOLECULAR CELLULAR INTRINSIC AND RESVERATROL}

\begin{abstract}
The increase of intracelular ROS induces oxidative stress, which can trigger cellular death by apoptosis, by the activation of intrinsic apoptotic cascades. Oxidative stress may occur in the organism in different situations and in various organs and tissues. The consumption of fat in the diet is associated with the increase of the oxidative stress and mitochondrial dysfunction in various organs. Antioxidant substances act by inhibiting the excessive production of reative oxygen species (ROS), eliminate freee radicals and reduce apoptosis. Resveratrol is a compound that can be found in plants and has been shown to have various biological properties, one of them is the antioxidant activity. The target of this review is to gather information about the molecular mechanisms of antioxidant action of the intrinsic cellular pathways and resveratrol in cells.
\end{abstract}

KEYWORDS: Diabetes, heart attack, ischemia. 


\section{INTRODUÇÃO}

A fisiopatologia de diversas doenças cardiovasculares e neuronais é complexa e tem participação fundamental do fenômeno conhecido por estresse oxidativo. O equilíbrio metabólico do oxigênio no meio intracelular é mantido por enzimas antioxidantes, também conhecidas como enzimas de fase II de desintoxicação, como a heme oxigenase 1 (HO-1). Essas proteínas atuam na regulação e supressão do estresse oxidativo em células endoteliais e neurais. A produção dessas enzimas ocorre por meio da ativação da via de sinalização antioxidante fator eritróide nuclear 2 relacionado ao fator 2 (Nrf2) / elemento de resposta antioxidante (ARE), por meio de diversas substâncias pesquisadas com potencial antioxidante (LEE et al., 2015; XIA et al., 2015).

As substâncias antioxidantes atuam inibindo a produção excedente de espécies reativas de oxigênio (ROS). Como exemplo, a isquemia e a reperfusão provocam lesão na mucosa intestinal por meio de metabólitos ROS derivados do sistema da xantina oxidase após a reperfusão. A glutamina protege o intestino inibindo a produção de ROS, a clivagem da caspases, apoptose e a peroxidação lipídica por meio da ativação da via de sinalização Nrf2/ARE. Por essa razão, a glutamina é uma substância que foi utilizada com sucesso no tratamento de lesões experimentais de isquemia e reperfusão no intestino de ratos (WANG et al., 2015).

A importância das lesões por acúmulo de ROS também pode ser ilustrada por episódios múltiplos e recorrentes de isquemia e reperfusão e isquemia miocárdica transitória, que resultam em significativos danos no miocárdio. Tais lesões incluem a morte de cardiomiócitos, fibrose e adelgaçamento da parede do coração, prejudicando a função ventricular e resultando em insuficiência cardíaca. Nesses casos, a enzima HO-1 previne o enfraquecimento da parede do miocárdio, a inflamação, a fibrose e a disfunção cardíaca induzida pelas lesões, com redução da morte celular por apoptose. Os mecanismos antioxidantes protegem os cardiomiócitos e podem ser uma estratégia de proteção para pacientes com doença arterial coronariana e com alto risco de isquemia miocárdica recorrente (PACHORI et al., 2006).

Com esta revisão de literatura pretendeu-se reunir informações sobre os mecanismos moleculares de ação antioxidante de vias celulares intrínsecas e do resveratrol nas células.

\section{ESTRESSE OXIDATIVO}

O aumento intracelular de ROS induz o estresse oxidativo, que pode desencadear a morte celular por apoptose, por ativação de cascatas apoptóticas intrínsecas. $O$ estresse oxidativo induzido por radicais livres pela clivagem das caspases quatro e 12 por tempo prolongado no retículo endoplasmático resulta em apoptose (VANI et al., 2013). O estresse oxidativo pode se apresentar no organismo em diferentes situações e nos mais diversos órgãos e tecidos (VANI et al., 2013).

As células submetidas à situação de isquemia e reperfusão (I/R) aumentam a produção de ROS e consequentemente induzem ao estresse oxidativo e a morte celular mediada pela tensão oxidativa. Na mucosa intestinal, a lesão decorrente da I/R é induzida pela produção de ROS por meio do sistema de xantina oxidase após a reperfusão (PACHORI et al., 2007; LEE et al., 2015 ).

O estresse oxidativo também pode ser um importante fator para o desenvolvimento de doenças do fígado. ROS formados tanto endogenamente como consequência do metabolismo de xenobióticos são eliminados por enzimas 
e por sistemas celulares não enzimáticos. O estresse oxidativo aumenta a liberação de lactato desidrogenase (LDH) e promove alterações histológicas como perda de integridade celular, vacuolização e degradação das células acometidas (RUBIOLO et al., 2008).

O consumo de dieta rica em gordura está relacionado com o aumento do estresse oxidativo e disfunções mitocondriais em diversos órgãos. Este promove o aumento da expressão de glicogênio sintase quinase 3 beta (GSK-3 beta) no sangue periférico e no baço de animais com consumo de dieta rica em gordura. Em contraste, o nível do Nrf2 em células T reguladoras (Treg) diminui, assim como a expressão de $\mathrm{HO}-1$ e da proteína de desacoplamento 2 (UCP2) (WANG et al., 2014).

\section{SUBSTÂNCIAS ANTIOXIDANTES}

As enzimas antioxidantes, também conhecidas como enzimas de fase II de desintoxicação, são proteínas que inibem a produção de ROS, eliminam radicais livres e consequentemente diminuem a apoptose induzida pelo estresse oxidativo. As principais enzimas são $\mathrm{HO}-1, \mathrm{NAD}(\mathrm{P}) \mathrm{H}$ : oxidorredutase quinina 1 (NQO1) e gama-glutamilcisteína sintase (gama-GCS) que são mediadas pela via de sinalização Nrf2/ARE (VANI et al., 2013).

Heme oxigenase-1 é uma enzima isoforma induzível cujo RNA mensageiro (RNAm) é transcrito a partir de um gene de resposta ao estresse oxidativo, sendo regulada por vários estímulos fisiológicos e exógenos. É responsável pela manutenção da homeostase redox e executa papel essencial na proteção contra o estresse oxidativo. Atua ainda na degradação do heme em biliverdina, que posteriormente é convertida em bilirrubina pela enzima biliverdina redutase. Tanto a biliverdina como a bilirrubina possuem também ação antioxidante. HO-1 apresenta atividade citoprotetora no pós-condicionamento isquêmico, um procedimento de interrupções rápidas e intermitentes do fluxo sanguíneo na fase inicial da reperfusão que pode proteger um órgão de lesão de I/R. Induz proteção contra isquemia cerebral transiente e permanente, atenuando o volume de infarto no cérebro, podendo ser fundamental na proteção contra acidente vascular cerebral. Em células endoteliais, HO-1 está associada à proteção contra lesão endotelial relacionada com estresse oxidativo vascular; por esse motivo, sua indução pode ser considerada uma intervenção terapêutica em doenças cardiovasculares (PACHORI et al., 2007; ZEYNALOV et al., 2009; ZENG et al., 2011; LEE et al., 2015).

Uma via enzimática de papel preponderante na proteção neural contra o estresse oxidativo é a Nrf2/ARE. Uma lesão medular promove a perda da estrutura e função dos axônios que não podem crescer ou regenerar espontaneamente após o dano, necessitando de tratamentos com neurotrofina. Em casos como esse, o fator de crescimento do nervo (NGF) promove o crescimento e a regeneração de novos axônios, que pode ocorrer por meio da ativação de vias de sinalização antioxidante Nrf2/ARE, mediada por Akt e associada ao NGF (XIA et al., 2015).

Amplamente conhecidas, a glutationa (GSH) e a superóxido dismutase (SOD) são enzimas antioxidantes que podem ser encontradas em diferentes localizações. A GSH pode ser encontrada no fígado em grandes quantidades; já a SOD1, também chamada de Cu/Zn-SOD, é localizada no citosol de diferentes células e a SOD2, também chamada de Mn-SOD, está localizada na mitocôndria. 
Estas inibem a produção de ROS e protegem as células dos danos induzidos pelo estresse oxidativo (KHAN et al., 2013; DAS et al., 2015).

Além dos mecanismos antioxidantes endógenos, há compostos com efeito similar que podem ser consumidos na dieta diária. O resveratrol, por exemplo, é um composto que ocorre naturalmente em plantas, como as uvas (baga e pele), frutas vermelhas e amendoim, assim como em vinhos tintos, fazendo, portanto, parte da dieta humana. É uma molécula que tem demonstrado possuir diversas propriedades biológicas, dentre elas a propriedade antioxidante. Antioxidantes exógenos também podem desempenhar importante papel na proteção contra o desenvolvimento de doenças que cursam com danos provocados por moléculas oxidativas (RUBIOLO et al., 2008; WANG et al., 2014; KUROYANAGI et al., 2015).

\section{Mecanismos de ação antioxidante}

\section{VIA NRF2/ARE}

A via de sinalização antioxidante Nrf2/ARE (figura 1) é indispensável para a indução da expressão de enzimas antioxidantes como a HO-1, gamaglutamilcisteína sintase (gama- GCS), NQO-1, SOD. O Nrf2 é ativado e este é translocado do citoplasma para o núcleo, após a degradação da proteína Kelch 1 associada a ECH (Keap-1) por proteassomas. Ao penetrar no núcleo, o Nrf2 se liga ao ARE promovendo então a indução do RNAm das enzimas antioxidantes, que agem promovendo a inibição do estresse oxidativo e a morte celular. A ativação de Nfr2 é mediada por substâncias antioxidantes, moléculas ativadoras como o Akt, Fosfatidilinositol 3-quinase (PI3K), entre outras (MIN et al., 2011; VANI et al., 2013; JEONG et al., 2014; XIA et al., 2015).

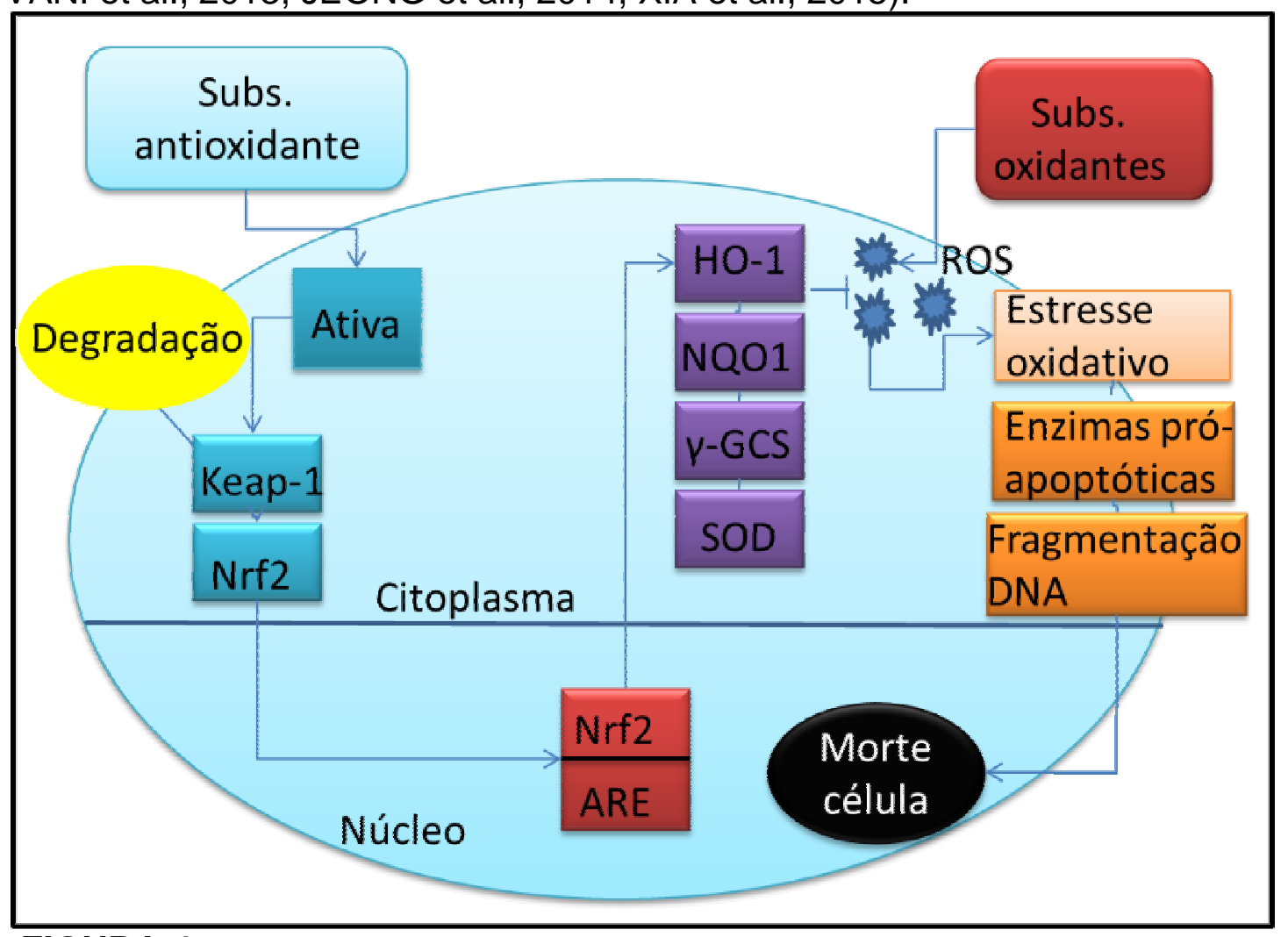

FIGURA 1 - Esquema representativo da via de transdução de sinal das enzimas fator eritróide nuclear 2 relacionado ao fator 2 (Nrf2) / elemento de resposta antioxidante (ARE).Fonte: Arquivo Pessoal, 2015. 
A linhagem celular PC12, originada de feocromocitoma de ratos, é um modelo de célula frequentemente utilizada em estudos de diferenciação e desenvolvimento neural. Essas células foram tratadas com fator de crescimento neuronal (NGF) e Akt, que promoveram o crescimento axonal, aumentaram a viabilidade celular e reduziram a apoptose. O Akt aumentou a expressão de enzimas antioxidantes HO-1, NQO1 e gama-GCS por meio da ativação da via Nrf2/ARE, principal mecanismo na prevenção do estresse oxidativo. O tratamento com Akt, associado ao NGF, contribui para a regeneração neuronal e pode ser uma promissora terapia para lesões medulares (XIA et al., 2015).

Em células cancerígenas de cólon humano da linhagem HT29, a proteína Bay 11-7082 (Bay) aumenta a expressão de RNAm de HO-1, por meio da ativação da via Nrf2/ARE, indispensável para a indução. Induz também a produção de ROS, a fosforilação de Akt e a expressão de $\mathrm{HO}-1$ por meio da via de sinalização PI3K/Akt. A proteína Bay induz a expressão de HO-1 por duas vias de sinalização diferentes e de forma independente da via Fator nuclear kappa beta (NF-kappa beta) (MIN et al., 2011).

Antcin $\mathrm{C}$ é um composto esteroide, isolado do cogumelo medicinal Antrodia cinnamomea, que possui ação antioxidante e protege as células do fígado, in vivo e in vitro, do estresse oxidativo e da morte celular pela ativação da via de sinalização Nrf2/ARE. Células hepáticas da linhagem hepatoma humano (HepG2) foram induzidas pela substância 2,2-azobis (2-amidinopropano) dihidrocloreto (AAPH) ao estresse oxidativo e a morte celular. $\mathrm{O}$ antcin $\mathrm{C}$ protegeu o fígado de lesões, o que foi comprovado pela constatação que os níveis de alanina aminotransferase (ALT) e aspartato aminotransferase (AST) se encontravam dentro dos padrões de referência. O composto testado inibiu a peroxidação lipídica e aumentou o nível de glutationa (GSH). Ainda, induziu os genes de substâncias antioxidantes, como gama-glutamil cisteina sintase subunidade catalítica (gama-GCLC), HO-1, NQO1 e SOD, assim como seu fator de transcrição correspondente Nfr2, aumentando significativamente os níveis de expressão dessas proteínas e da célula-B de linfoma 2 (Bcl2). A ativação de Nfr2 é mediada pela fosforilação de c-Jun N-terminal quinase 1/2 (JNK1/2), um membro da família da proteína quinase ativada por mitógeno (MAPK), ou pode ser mediado também pela PI3K. A substância também inibiu o citocromo $\mathrm{c}$, a clivagem das caspases 3 e 9, o Poli [ADP ribose] polimerase (PARP) e a Bcl2 do tipo 4 (Bax), que são mediadores pró-apoptóticos. Antcin $C$ inibiu a clivagem das caspases $4 \mathrm{e}$ 12 , impedindo o estresse oxidativo no retículo endoplasmático que resultaria em apoptose e regula positivamente o gene protetor HSP70 contra o estresse oxidativo no reticulo endoplasmático. $\mathrm{O}$ antcin $\mathrm{C}$ in vivo tem efeito citoprotetor com regulação positiva de antioxidantes, protegendo as células hepáticas de estresse oxidativo por meio da ativação da via Nrf2 / ARE. Por fim, esse composto promoveu aumento da produção de GSH, inibiu a peroxidação lipídica e suprimiu a apoptose induzida por radicais livres por meio da supressão da geração de ROS (VANI et al., 2013).

Arctigenina é uma lignina, a dibenzilbutirolactona fenilpropanóide, isolada a partir das sementes de Arctium lappa. Esta substância tem potencial terapêutico para uma variedade de doenças neurodegenerativas que cursam com o estresse oxidativo, pois tem ação antioxidante. Em astrócitos de ratos de cultivo primário, a arctigenina modula a via de sinalização Nfr2/ARE por meio da via PI3K/Akt, que está parcialmente envolvida na ativação da via Nrf2/ARE e por Junc, aumentando a expressão do RNAm de HO-1 (JEONG et al., 2014). 
O composto C (6- [4- (2-piperidina-1-il-etoxi) -fenil] -3-piridina-4-ilpirazolo [1,5-a] pirimidina) é um potente inibidor de proteína quinase adenosinamonofosfato ativada (AMPK) por meio da competição com adenosina trifosfato (ATP). O composto estimulou a expressão de $\mathrm{HO}-1$ em células endoteliais vasculares humanas por ativação da via Nrf2/ARE. A glutationa, doadora de Nacetil-L-citeína, bloqueou o aumento na formação de ROS. A bilirrubina e a biliverdina são produtos de $\mathrm{HO}-1$ que mediam o efeito citoprotetor do composto $\mathrm{C}$ (LIU et al., 2011).

Lignanas foram isoladas da fruta Schisandra chinensis, utilizada no Sudeste da Ásia. Essas substâncias contêm o composto gomisina, que regula a expressão de moléculas inflamatórias e tem ação antioxidante em micróglias (figura 2). Em células microgliais foi induzida inflamação com posterior tratamento com gomisina. Este teve ação antioxidante, pois inibiu a produção de ROS e ativou MAPK e a ativador Janus quinase (JAK)/ transdutor de transcrição Janus quinase (STAT). Inibiu também a expressão de moléculas inflamatórias como óxido nítrico, prostaglandina E2, citocinas (fator de necrose tumoral-alfa (TNFalfa), interleucina-1beta (IL-1beta), interleucina 6 (IL-6)) e quimiocina (quimiocina quimioatrativa de monócitos da proteína-1 - MCP-1) por meio do bloqueio da ativação da via NF-kappa beta. Aumentou os níveis de AMP cíclico (AMPc) e a fosforilação de proteína de ligação do elemento (CREB). As principais enzimas envolvidas na sinalização de Nrf2, proteína quinase (PKA) e CREB, tiveram os níveis aumentados. A gomisina também aumentou a translocação do Nrf2 para o núcleo e a indução da expressão das enzimas HO-1 e NQO1 (PARK et al., 2014).

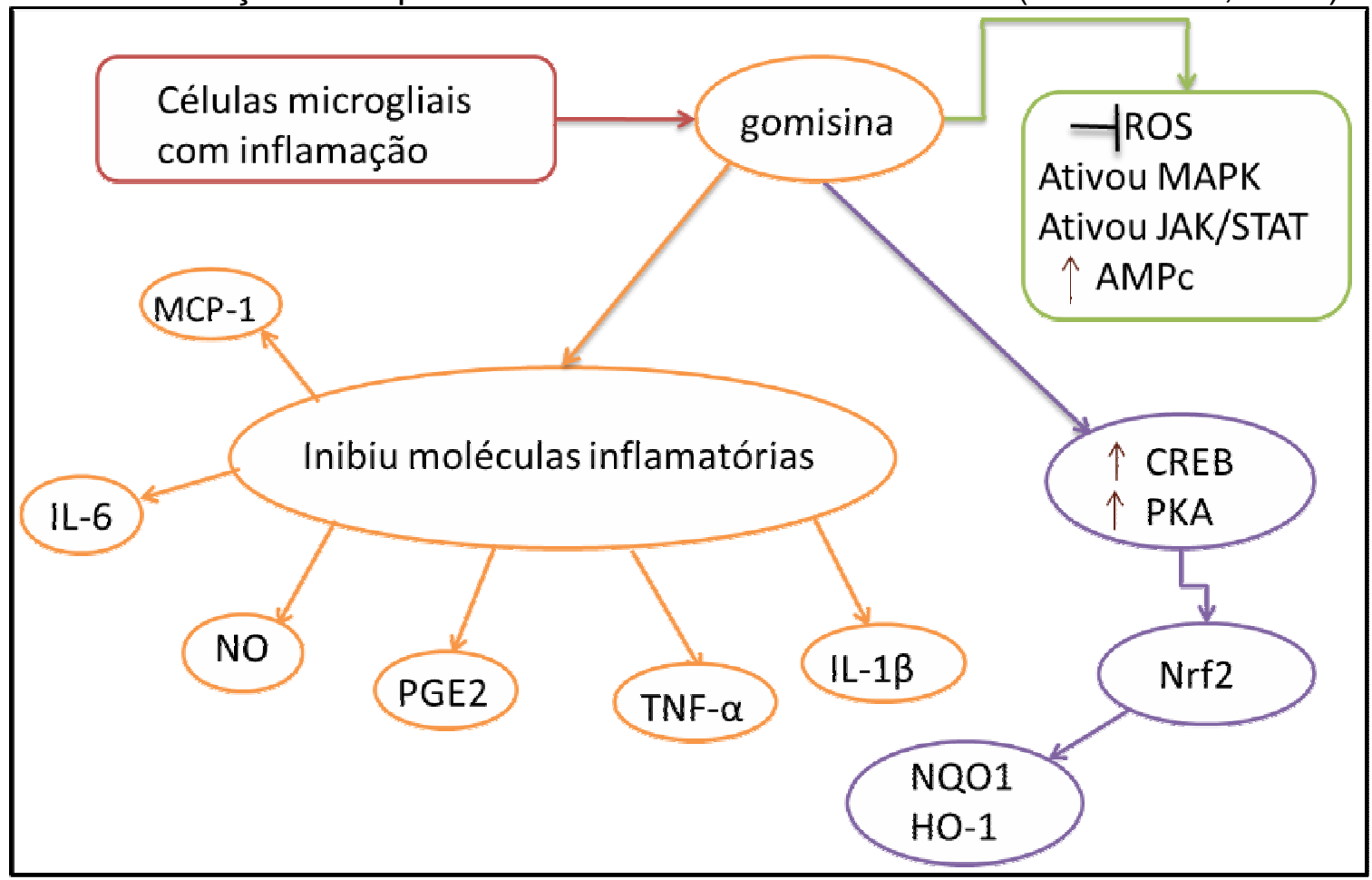

FIGURA 2 - Esquema representativo da via de ação anti-inflamatória e antioxidante da gomisina em células microgliais. Fonte: Arquivo Pessoal, 2015.

Durante o procedimento experimental de transplante de fígado em ratos foi realizado o pós-condicionamento isquêmico. A enzima $\mathrm{HO}-1$ foi expressa predominantemente no citoplasma das células de Kupffer, tendo induzido efeito citoprotetor contra a lesão de isquemia e reperfusão hepática e aumentado a 
atividade antioxidante. Os efeitos protetores do pós-condicionamento isquêmico foram significativamente associados com maior expressão intra-hepática de HO-1. Houve aumento das atividades da enzima SOD e redução da atividade da peroxidação lipídica, indicando a indução de mecanismos endógenos citoprotetores (ZENG et al., 2011).

Indol-3-carbinol, fenetilo isotiocianato, ácido protocatecuico e ácido tânico são fitoquímicos que ocorrem naturalmente e fazem parte da dieta diária humana. Estas substâncias aumentam o nível de atividade de enzimas de fase II e proteínas hepáticas em modelos animais. Em células de carcinoma hepatocelular humano (HepG2), essas substâncias promoveram a translocação do citosol e acumulação nuclear da forma ativa (fosforilada) de Nrf2. Além disso, aumentaram a transcrição e os níveis de proteína de isoforma da glutationa S-transferase (GSTA, GSTM, GSTT), receptor-gama coactivador-1alfa (SPGC) e NQO1 A expressão de genes que codificam as enzimas antioxidantes catalase (CAT), SOD, glutationa redutase (GR), e glutationa peroxidase (GPx) foi aumentada após o tratamento com todos os fitoquímicos testados por meio da via de sinalização Nrf2/ARE com interação com a proteína Keap-1. A proteína acumulada foi Nrf2 e fosforilada em Ser 40 no núcleo depois de exposição a todos os fitoquímicos estudados. A atividade contra lesões por substâncias tóxicas e/ou oxidantes comprovam sua propriedade antioxidante (KRAJKA-KUZNIAK et al., 2015).

Em células endoteliais, o eridictiol induziu aumento da expressão do RNAm e da proteína HO-1 por meio da sinalização de quinase regulada extracelularmente (ERK) e da ativação da via Nrf2/ARE. Esta substância teve efeito protetor contra o estresse oxidativo nas células endoteliais, reduzindo a produção de ROS pela regulação positiva da enzima HO-1 (LEE et al., 2015).

Ratos foram submetidos à isquemia e reperfusão intestinal e posteriormente tratados com glutamina. A glutamina reduziu a lesão do jejuno e a permeabilidade microvascular por meio do aumento dos níveis de ativação da via Nrf2/ARE e da expressão de HO-1. Nas células do jejuno também ocorreu aumento dos níveis de Bcl-2 e diminuição do nível da caspase 3, promovendo supressão da apoptose. O aumento do nível de Bcl-2 ocorreu por meio da via Nrf2/ARE. A produção de ROS foi inibida e a apoptose intestinal foi reduzida. A redução do estresse oxidativo resultou em aumento da atividade da SOD e redução da peroxidação lipídica induzida pela glutamina (WANG et al., 2015).

\section{VIA PI3K/AKT}

$\mathrm{Na}$ diabete, o elevado teor de glicose induz a superprodução de ROS. Estes ativam a via de sinalização PI3K/Akt, que ativam por sua vez a via alvo da rapamicina em mamíferos (mTOR)/ p70 ribossomal S6 proteina quinase (p70S6K) em células neuronais HT-22 e nos cérebros de ratos diabéticos. A ativação da via mTOR/p70S6K induz ao estresse oxidativo e eleva a expressão da enzima acetilcolinesterase (LIU et al., 2015).

Em células $\mathrm{H} 9 \mathrm{c} 2$, linhagem celular de cardiomiócitos foram induzidas a isquemia e reperfusão, o que resultou em morte celular por apoptose por meio do aumento do estresse oxidativo e da atividade das caspases. A enzima HO-1 desempenhou papel protetor nas células por meio da inibição da apoptose e do estresse oxidativo. O mecanismo de ação desta enzima foi mediado pela enzima biliverdina redutase e pela via de sobrevivência celular PI3K/Akt. HO-1 diminui a atividade das caspases e a via PI3K/Akt inibe as vias apoptóticas nas mitocôndrias. O aumento da atividade de Akt foi resultante da sua fosforilação pela 
proteína fosfatidilinositol 3-quinase subunidade alfa ( $\mathrm{p85}$ ), mediado pela enzima HO-1 e parcialmente pela enzima biliverdina redutase. A ativação de Akt por $\mathrm{HO}-1$ é realizada a nível pós-transducional por vários mecanismos e possivelmente inclui eventos diretos de p85 e Akt por HO-1 ou pela enzima biliverdina redutase ou ambos (PACHORI et al., 2007).

\section{RESVERATROL: UM ANTIOXIDANTE EXÓGENO}

O resveratrol (Res) promove a depleção de ATP, condição que ativa a AMPK. Esta reduz os níveis de ROS intracelular e está envolvida na regulação positiva ao nível transcricional, aumentando a expressão das enzimas antioxidantes manganês superóxido dismutase (MnSOD) e catalase (CAT). O fator de transcrição forhead (FoxO1) desempenha um papel fundamental na expressão do gene MnSOD e CAT em resposta ao resveratrol. A transcrição de FoxO1 é mediada pela AMPK, que pode fosforilar FoxO1 diretamente. Após a fosforilação da FoxO1 no receptor Thr649 pela AMPK, ocorre a translocação da primeira para o núcleo, impedindo que enzima sofra degradação proteossômica e fique estável no núcleo. Em síntese, Os efeitos antioxidantes do resveratrol ocorrem pela ativação da AMPK, que fosforila o fator de transcrição FoxO1 (YUN et al., 2014).

O composto dapsona hidroxilamina (DDS) é um dos fármacos recomendados pela Organização Mundial de Saúde para o tratamento da hanseníase. No entanto, o DDS produz metabólitos que causam nos pacientes efeitos adversos ligados ao estresse oxidativo, como a formação de metahemoglobina e danos no ácido desoxirribonucleico (DNA). Em linfócitos e eritrócitos humanos, o Res inibiu os danos no DNA, bem como a formação de ROS pela atividade da enzima CAT. Com isso, o pré-tratamento com Res durante a terapia com DDS pode diminuir a oxidação de ferro heme e os danos no DNA pela redução da produção de ROS, mas não reverte completamente a formação da metahemoglobinemia, como observado no tratamento com azul de metileno (MET)(ALBUQUERQUE et al., 2015).

O MET é potencialmente melhor antioxidante quando comparado com o Res, pois o MET possui mais grupos doadores de elétrons que o Res. A nucleofilicidade de ambos os medicamentos pode ser expressa pela ionização do valor potencial (IP), que é calculado como a energia necessária para a captação de um elétron na molécula. IP representa a facilidade de doação de elétrons nestas moléculas, sendo o mecanismo primário antioxidante. A elevada atividade de reversão de metahemoglobinemia está associada com menor valor de IP. Com isso, as moléculas com valores baixos de IP podem sofrer oxidação com maior facilidade. A energia de estabilização relaciona os valores de energia para a reatividade química através da redução de um elétron entre duas moléculas diferente, entre DDS e Res ou entre DDS e MET. Res tem menor energia de estabilização que o MET. Portanto, Res tem menor capacidade de reverter a metahemoglobinemia que MET (ALBUQUERQUE et al., 2015).

Em células cancerígenas como de câncer de próstata (PC-3), hepatoma humano (HepG2) e câncer de mama (MCF-7), o Res tem efeito pró-apoptótico, com acúmulo de peróxido de hidrogênio nas células, induzindo ao estresse oxidativo e morte celular. Porém, este efeito não ocorre em células do câncer de mama. A regulação das enzimas antioxidantes nessas células, bem como a ação enzimática, ocorre de forma desproporcional, sendo a expressão da SOD1 aumentada e a expressão da CAT e GPx diminuída. Esta desproporção resulta em acúmulo do peróxido de hidrogênio na mitocôndria. $O$ efeito do Res ocorre apenas 
em algumas células cancerígenas. Na linhagem celular HEK293T, nãocancerígena, o Res não provocou aumento da taxa de apoptose. No entanto, a uma concentração mais elevada, Res induziu efeito inibitório do crescimento dessas células não-cancerígenas, sugerindo efeito colateral da substância com a utilização de doses mais elevadas (KHAN et al., 2013).

A sobrecarga de ferro hepático pode ser incrementada pela hemocromatose genética e está associada com hepatoesplenomegalia, aumento do estresse oxidativo, fibrose hepática, inflamação e indução pró-apoptótica. Esses efeitos foram revertidos com terapia com Res. A sobrecarga de ferro hepático crônica provocou aumento de localização nuclear de acetilação do fator de transcrição FoxO1, que foi revertida pela intervenção dietética com Res em associação com níveis aumentados de sirtuína 1 (SIRT1). O Res não afetou o grau de sobrecarga de ferro hepático, mas protegeu o fígado da lesão provocada pelo ferro, ativou SIRT1 e modulou a desacetilação de FoxO1, que por sua vez induziu resposta antioxidante durante 0 estresse oxidativo. $O$ aumento da acetilação de FoxO1 é o principal mecanismo na patogênese da sobrecarga hepática, que foi revertido pelo Res. Durante o estresse oxidativo, há presença de FoxO1 no núcleo das células induzidos pelo ferro e baixo níveis de SIRT1 (DAS et al., 2015).

O estresse oxidativo provocado pela sobrecarga de ferro resultou na peroxidação lipídica, aumento de radicais livres, reduziu os níveis de glutationa reduzida, aumentou a glutationa oxidada e reduziu drasticamente a proporção da reduzida em relação a oxidada, tendo aumentado ainda a geração de malondialdeído (MDA), um produto da peroxidação lipídica. Suprimiu também a expressão dos genes antioxidantes CAT e SOD1. Res, que tem potente efeito antioxidante no fígado, aumentou a reserva de glutationa, reduziu a peroxidação lipídica e normalizou a expressão dos genes antioxidantes da CAT e SOD1. A sobrecarga de ferro está associada ao aumento de infiltração por neutrófilos, macrófagos linfócitos CD-8 positivas, aumento da expressão de citocinas próinflamatórias, IL-6, IL-1beta, TNF-alfa e da caspase-3 clivada. Res reduziu significativamente a inflamação e a morte celular por apoptose (DAS et al., 2015).

No fígado, o aumento da ativação oxidativa e do metabolismo lipídico podem resultar em esteatose hepática. Após a suplementação com Res, os níveis de ROS foram diminuídos e a indução de estresse oxidativo inibido. Os níveis séricos de triglicerídeos e MDA diminuíram, enquanto os níveis de lipoproteína de alta densidade (HDL-C) foram aumentados. Aumentaram também os níveis de GSH e da GPx e SOD. A expressão das proteínas de membrana p47phox e gp91phox diminuiu, enquanto a expressão das proteínas p-HSL, hormônio sensível a lipase (HSL) e lipase triglicerídeos adiposos (ATGL) aumentou. Aumentaram os níveis de SIRT1 e a fosforilação da AMPK, o que provocou a desacetilação da FoxO1. Res demonstrou poder atenuar a esteatose hepática e o distúrbio metabólico lipídico além de melhorar a capacidade antioxidante em camundongos KKAy, sugerindo que pode ser utilizado para tratamento de esteatose hepática no fígado humano (ZHU et al., 2014).

O estresse oxidativo é um importante fator para o desenvolvimento de doenças no fígado. ROS gerados endogenamente ou como consequência do metabolismo de xenobióticos são eliminados por enzimas e por sistemas celulares não enzimáticos. Os danos oxidativos podem ser prevenidos por antioxidantes endógenos e por meio da dieta. Nos hepatócitos primários em cultura, induzidos ao estresse oxidativo, Res aumentou a atividade das enzimas catalase, 
superóxido dismutase, glutationa peroxidase, NQO1 e glutationa-S-transferase. Aumentou também o nível de Nrf2 e a concentração de RNAm. Res inibiu parcialmente a morte celular por necrose induzida pelo estresse oxidativo, além de proteger as células, inibindo a perda da integridade celular, vacuolização celular e degradação e também verificou-se que impediu a atividade da caspase 3 e consequentemente a apoptose. A ativação do Nrf2 pelo Res aumenta a expressão dos genes de transcrição das enzimas de fase II NQO1 e NAD (P) H: oxidorredutase quinina 2 (NQO2) e as atividades enzimáticas das mesmas. O Res poderia ser usado como uma proteção contra o estresse oxidativo no fígado humano (RUBIOLO et al., 2008).

A síntese de proteínas como mTOR e p70S6 quinase contribuem para a hipertrofia ventricular esquerda. Essas proteínas são moduladas por vias incluindo o quinase hepática B1 (LKB1)/AMPK. O estresse oxidativo inibe essa via de sinalização e promove o crescimento celular anormal de células cancerígenas. $A$ hipertrofia ventricular esquerda está associada com um aumento da peroxidação lipídica. Res impede a inibição da via de sinalização LKB1/AMPK e atenua a peroxidação lipídica e a ativação da via mTOR/p70S6. Res inibe o desenvolvimento da hipertrofia ventricular esquerda, sugerindo que seja uma terapia potencial para os pacientes em risco de desenvolver essa doença, pois promove melhora no desempenho cardíaco (DOLINSKY et al., 2009).

Nas células MC3T3-E1 tipo osteoblasto Res suprimiu significativamente a liberação do fator de crescimento endotelial vascular (VEGF) estimulado pelo fator de crescimento transformante-beta (TGF-beta) e os níveis de expressão de RNAm de VEGF. SRT1720, um ativador sintético de SIRT1, também reduziu a liberação de VEGF e os níveis de RNAm. RES e SRT1720 atenuaram significativamente a fosforilação de p44 / p42 MAPK e de SAPK/JNK estimulada por TGF-beta. A síntese de VEGF estimulada por TGF-beta é suprimida pelo RES através da inibição de MAPK p44/p42 e de SAPK/JNK em osteoblastos. O efeito supressor é mediado em parte pela ativação da SIRT1. O efeito supressor de Res na síntese de VEGF estimulada por TGF-beta é exercido provavelmente a montante do nível de transcrição em células MC3T3-E1 semelhantes a osteoblastos (KUROYANAGI et al., 2015).

O consumo de dieta rica em gordura (high-fat diet - HFD) está relacionado com o aumento do estresse oxidativo e disfunções mitocondriais em muitos órgãos. Res diminuiu significativamente a morte induzida por HFD nas células Treg, sendo associada com a redução da produção de ROS e da perda do potencial de membrana mitocondrial. Aumentou a expressão de fatores que regulam a biogênese mitocondrial de Treg, além de ter protegido as células Treg contra a apoptose induzida por HFD, reduzindo o estresse oxidativo, restaurando atividades funcionais mitocondriais. FoxP3, CTLA4 e fator de crescimento transformante-beta (TGF-p) aumentou significativamente no sangue periférico e Treg esplênicas após tratamento com Res. No sangue periférico e no baço de ratos induzidos HFD a expressão de GSK-3 beta aumentou significativamente. Em contraste, diminuiu o nível do Nrf2 significativamente e a expressão de HO-1 e UPC2. Res diminuiu o regulador da via pró-apoptótica mitocondrial Bax no baço. $\mathrm{O}$ nível de Bcl2, uma proteína anti-apoptótica, aumentou significativamente, enquanto diminuiu o nível da caspase 3 (WANG et al., 2014). 


\section{CONSIDERAÇÕES FINAIS}

O oxigênio é um elemento essencial à vida. Seja na forma de gás ou combinado a dois átomos de hidrogênio para formar a água, representa juntamente com o carbono o elemento primordial da existência dos seres vivos. Reações químicas diametralmente opostas, a fotossíntese e a respiração aeróbica, constituem a base das relações metabólicas e de interdependência entre todos os indivíduos que habitam a Terra. Por tudo isso, parece paradoxal o conceito de que o oxigênio pode provocar a destruição da célula que tanto depende desse mesmo elemento.

O estresse oxidativo é um componente metabólico cotidiano das células que fazem respiração aeróbica. Esses radicais, altamente eletronegativos, possuem elevado poder de ligação às diversas estruturas celulares, provocando alterações em sua estrutura e consequente modificação de suas propriedades biológicas. Um exemplo significativo é o dano que pode ser causado ao DNA; espécies reativas de oxigênio podem provocar mutações no código genético, silenciando proteínas importantes ou induzindo a síntese de proteínas indesejáveis, o que pode acarretar em morte da própria célula via apoptose, ou ainda mais grave, ao surgimento de um grupo de células de crescimento incontrolado, independente e indefinido, denominadas de células neoplásicas.

Para reduzir a possibilidade que a célula ou o indivíduo sucumbissem a esse processo inevitável, mecanismos evolutivos de controle foram desenvolvidos para permitir a sobrevivência dos seres vivos diante da ameaça promovida pelos radicais livres de oxigênio gerados pelo metabolismo celular. Dessa forma, vias de transdução de sinal como as das proteínas HO-1, NQO1 e gama-glutamilcisteína sintase, mediadas pela via de sinalização Nrf2/ARE, vêm contribuindo para a sobrevivência das espécies ao minimizarem os efeitos deletérios do estresse oxidativo sobre as células.

Além dos mecanismos celulares antioxidantes que se desenvolveram evolutivamente, a capacidade observadora da espécie humana e sua capacidade de compreensão dos fenômenos têm permitido que a espécie usufrua, de forma consciente, de antioxidantes presentes na natureza, mormente em vegetais. $O$ resveratrol tem sido apontado como um poderoso antioxidante natural, cuja epifania surgiu a partir da constatação do famoso "paradoxo francês", ou seja, em um país onde o elevado consumo de carnes e molhos repletos de gordura, os índices de doença cardiovascular são relativamente pouco significativos! Essa aparente incoerência foi atribuída ao elevado consumo per capita de vinho tinto, que contém o resveratrol, poderosa molécula antioxidante.

A partir do reconhecimento científico da ação benéfica do resveratrol como antioxidante, todo um horizonte de pesquisa de novos princípios com características similares presentes em plantas se descortina. Um bioma com elevado potencial para a existência de plantas que contenham antioxidantes naturais é o cerrado. As plantas da região suportam vários meses ao ano de sol inclemente e baixa umidade, condições inóspitas que se adaptaram ao longo de milhões de anos de evolução. Assim, plantas como o pequi, o ipê, a gabiroba, entre outras, que possuem potencial antioxidante já comprovado in vitro, podem, em um futuro próximo, se constituirem em alternativas regionais sustentáveis para terapias naturais de controle dos efeitos deletérios do estresse oxidativo. 


\section{REFERÊNCIAS}

ALBUQUERQUE, R. V.; MALCHER, N. S.; AMADO, L. L.; COLEMAN, M. D.; DOS SANTOS, D. C.; BORGES, R. S.; VALENTE, S. A. S.; VALENTE, V. C.; MONTEIRO, M. C. In Vitro Protective Effect and Antioxidant Mechanism of Resveratrol Induced by Dapsone Hydroxylamine in Human Cells. PLoS One, v. 10, n. 8, p. 0134768, 2015. Disponível em: <http://journals.plos.org/plosone/article?id=10.1371/journal.pone.0134768>. doi:10.1371/journal.pone.0134768

DAS, S. K.; DESAULNIERS, J.; DYCK, J. R.; KASSIRI, Z.; OUDIT, G. Y. Resveratrol mediates therapeutic hepatic effects in acquired and genetic murine models of ironoverload. Liver International, 2015. Disponível em:

<onlinelibrary.wiley.com/doi/10.1111/liv.12893/suppinfo>. DOI:10.1111/liv.12893

DOLINSKY, V. W.; CHAN, A. Y.; ROBILLARD FRAYNE, I.; LIGHT, P. E.; DES ROSIERS C.; DYCK, J. R. Resveratrol prevents the prohypertrophic effects of oxidative stress on LKB1. Circulation, v. 119, n. 12, p. 1643-52, 2009. Disponível em: <http://circ.ahajournals.org>. doi: 10.1161/circulationaha.108.787440

JEONG, Y. H.; PARK, J. S.; KIM, D. H.; KIM, H. S. Arctigenin Increases Hemeoxygenase-1 Gene Expression by Modulating PI3K/AKT Signaling Pathway in Rat Primary Astrocytes. Biomolecules and Therapeutics (Seoul), v. 22, n. 6, p. 497502, 2014. Disponível em: <http://dx.doi.org/10.4062/biomolther.2014.121>. doi:10.4062

KHAN, M. A.; CHEN, H. C.; WAN, X. X.; TANIA, M.; XU, A. H.; CHEN, F. Z.; ZHANG, D. Z. Regulatory effects of resveratrol on antioxidant enzymes: a mechanism of growth inhibition and apoptosis induction in cancer cells. Molecules and Cells, v. 35, n. 3, p. 219-25, 2013. Disponível em: <http://molcells.org>. DOI/10.1007/s10059013-2259-z

KRAJKA-KUZNIAK, V.; PALUSZCZAK, J.; SZAEFER, H.; BAER-DUBOWSKA, W. The activation of the Nrf2/ARE pathway in HepG2 hepatoma cells by phytochemicals and subsequent modulation of phase II and antioxidant enzyme expression. Journal of Physiology and Biochemistry, v. 71, n. 2, p. 227-38, 2015. Disponível em: < http://link.springer.com/article/10.1007\%2Fs13105-015-0401-4>. doi 10.1007/s13105-015-0401-4

KUROYANAGI, G.; OTSUKA, T.; YAMAMOTO, N.; MATSUSHIMA-NISHIWAKI, R.; KOZAWA, O.; TOKUDA, H. Resveratrol suppresses TGF-beta-induced VEGF synthesis in osteoblasts: Inhibition of the p44/p42 MAPKs and SAPK/JNK pathways. Experimental and Therapeutic Medicine, v. 9, n. 6, p. 2303-10, 2015. Disponível em: <http://www.ncbi.nlm.nih.gov/pmc/articles/PMC4473361>.

Doi: 10.3892/etm.2015.2389

LEE, S. E.; YANG, H.; SON, G. W.; PARK, H. R.; PARK, C. S.; JIN, Y. H.; PARK, Y. $S$. Eriodictyol Protects Endothelial Cells against Oxidative Stress-Induced Cell Death through Modulating ERK/Nrf2/ARE-Dependent Heme Oxygenase-1 Expression. International Journal of Molecular Sciences, v. 16, n. 7, p. 14526-39, 2015. Disponível em: <www.mdpi.com/journal/ijms>. doi: 10.3390/160714526 
LIU, Y. W.; ZHANG, L.; LI, Y.; CHENG, Y. Q.; ZHU, X.; ZHANG, F.; YIN, X. X. Activation of mTOR signaling mediates the increased expression of AChE in high glucose condition: in vitro and in vivo evidences. Molecular Neurobiology, 2015. Disponível em: < http://link.springer.com/article/10.1007\%2Fs12035-015-9425-6>. doi: 10.1007/s12035-015-9425-6

LIU, X. M.; PEYTON, K. J.; SHEBIB, A. R.; WANG, H.; DURANTE, W. Compound C stimulates heme oxygenase-1 gene expression via the Nrf2-ARE pathway to preserve human endothelial cell survival. Biochemical Pharmacology, v. 82, n. 4, p. 371-9, 2011. Disponível em: <journal home page: www.elsevier.com/locate/biochempharm>. doi:10.1016/j.bcp.2011.05.016

MIN, K. J.; LEE, J. T.; JOE, E. H.; KWON, T. K. An IkappaBalpha phosphorylation inhibitor induces heme oxygenase-1(HO-1) expression through the activation of reactive oxygen species (ROS)-Nrf2-ARE signaling and ROS-PI3K/Akt signaling in an NF-kappaB-independent mechanism. Cellular Signalling, v. 23, n. 9, p. 1505-13, 2011. Disponível em: <journal homepage: www.elsevier.com/locate/cel I s ig>. doi:10.1016/j.cellsig.2011.05.013

PACHORI, A. S.; MELO, L. G.; ZHANG, L.; SOLOMON, S. D.; DZAU, V. J. Chronic recurrent myocardial ischemic injury is significantly attenuated by pre-emptive adenoassociated virus heme oxygenase-1 gene delivery. Journal of the American College of Cardiology, v. 47, n. 3, p. 635-43, 2006. Disponível em: <http://ac.elscdn.com/S073510970502629X/1>. doi:10.1016/2005.09.038

PACHORI, A. S.; SMITH, A.; MCDONALD, P.; ZHANG, L.; DZAU, V. J.; MELO, L. G. Heme-oxygenase-1-induced protection against hypoxia/reoxygenation is dependent on biliverdin reductase and its interaction with PI3K/Akt pathway. Journal of Molecular Cellular Cardiology, v. 43, n. 5, p. 580-92, 2007. Disponível em: <www.elsevier.com/locate/yjmcc>.doi:10.1016/j.yjmcc.2007.08.003

PARK, S. Y.; BAE, Y. S.; KO, M. J.; LEE, S. J.; CHOI, Y. W. Comparison of antiinflammatory potential of four different dibenzocyclooctadiene lignans in microglia; action via activation of PKA and Nrf-2 signaling and inhibition of MAPK/STAT/NFkappaB pathways. Molecular Nutrition \& Food Research, v. 58, n. 4, p. 738-48, 2014. Disponível em: <www.mnf-journal.com>. doi 10.1002/mnfr.201300445

RUBIOLO, J. A.; MITHIEUX, G.; VEJA, F. V. Resveratrol protects primary rat hepatocytes against oxidative stress damage: activation of the Nrf2 transcription factor and augmented activities of antioxidant enzymes. European Journal Pharmacology, v. 591, n. 1-3, p. 66-72, 2008. Disponível em <www. e Isevi e r.com/locate/ejphar>. doi:10.1016/j.ejphar.2008.06.067

VANI, M. G.; KUMAR, K. J.; LIAO, J. W.; CHIEN, S. C.; MAU, J. L.; CHIANG, S. S.; LIN, C. C.; KUO, Y. H.; WANG, S. Y. Antcin C from Antrodia cinnamomea Protects Liver Cells Against Free Radical-Induced Oxidative Stress and Apoptosis In Vitro and In Vivo through Nrf2-Dependent Mechanism. Evidence-Based Complementary Alternative Medicine, v. 2013, p. 296082, 2013. Disponível em: <http://dx.doi.org/10.1155/2013/296082. doi: 10.1155/2013/296082 
WANG, A. L.; NIU, Q.; SHI, N.; WANG, J.; JIA, X. F.; LIAN, H. F.; LIU, Z.; LIU, C. H. Glutamine ameliorates intestinal ischemia-reperfusion Injury in rats by activating the Nrf2/Are signaling pathway. International Journal Clinical and Experimental Pathology, v. 8, n. 7 , p. 7896-904, 2015. Disponivel em: <http://www.ncbi.nlm.nih.gov/pmc/articles/PMC4555682/pdf/ijcep0008-7896.pdf>. ISSN:1936-2625

WANG, B.; SUN, J.; MA, Y.; WU, G.; TIAN, Y.; SHI, Y.; LE, G. Resveratrol preserves mitochondrial function, stimulates mitochondrial biogenesis, and attenuates oxidative stress in regulatory T cells of mice fed a high-fat diet. Journal of Food Science, v. 79, n. $9, \quad$ p. 1823-31, 2014. Disponível em <http://onlinelibrary.wiley.com/doi/10.1111/1750-3841.12555/epdf>. doi: $10.1111 / 1750-3841.12555$

XIA, B.; LIU, H.; XIE, J.; WU, R.; LI, Y. Akt enhances nerve growth factor-induced axon growth via activating the Nrf2/ARE pathway. International Journal of Molecular Medicine, v. 35, n. 5, p. 1426-32, 2015. Disponível em: <http://www.ncbi.nlm.nih.gov/pubmed/26324295>. doi: 10.3892/2015.2329

YUN, H.; PARK, S.; KIM, M. J.; YANG, W. K.; IM, D. U.; YANG, K. R.; HONG, J.; CHOE, W.; KANG, I.; KIM, S. S.; HA, J. AMP-activated protein kinase mediates the antioxidant effects of resveratrol through regulation of the transcription factor FoxO1. The FEBS Journal, v. 281, n. 19, p. 4421-38, 2014. Disponível em: <http://onlinelibrary.wiley.com/doi/10.1111/febs.12949/abstract>.

doi:10.1111/febs.12949

ZENG, Z.; HUANG, H. F.; CHEN, M. Q.; SONG, F.; ZHANG, Y. J. Contributions of heme oxygenase-1 in postconditioning-protected ischemia-reperfusion injury in rat liver transplantation. Transplantation Proceedings, v. 43, n. 7, p. 2517-23, 2011. Disponível em: <http://ac.els-cdn.com/S0041134511008591/1-s2.0S0041134511008591>. doi:10.1016/j.transproceed.2011.04.021

ZEYNALOV, E.; SHAH, Z. A.; LI, R. C.; DORE, S. Heme oxygenase 1 is associated with ischemic preconditioning-induced protection against brain ischemia. Neurobiology of Disease, v. 35, n. 2, p. 264-9, 2009. Disponível em: <journal homepage: www.elsevier.com/locate/ynbdi>. doi:10.1016/j.nbd.2009.05.010

ZHU, W.; CHEN, S.; LI, Z.; ZHAO, X.; LI, W.; SUN, Y.; ZHANG, Z.; LING, W.; FENG, $X$. Effects and mechanisms of resveratrol on the amelioration of oxidative stress and hepatic steatosis in KKAy mice. Nutrition and Metabolism (Lond), v. 11, p. 35, 2014. Disponível em: < http://www.nutritionandmetabolism.com/content/11/1/35>. 